\title{
Correlação entre o ganho de peso e a intensidade da dor lombar em gestantes*
}

\section{Correlation between weight gain and low back pain intensity in pregnant women}

\author{
Cynthia Maria de Sousa Barbosaㄹ ${ }^{1}$, José Mário Nunes da Silva², Adeildes Bezerra de Moura \\ * Recebido da Faculdade Integral Diferencial (FACID). Teresina, PI.
}

\section{RESUMO}

JUSTIFICATIVA E OBJETIVOS: No período gestacional ocorre um aumento do aporte energético para satisfazer as necessidades da mãe e do feto, sendo a dor lombar um sintoma característico deste período. O objetivo deste estudo foi correlacionar o ganho de peso ponderal durante a gestação e a intensidade da dor lombar das gestantes.

MÉTODO: Realizou-se um questionário direcionado às gestantes contendo dados sociodemográficos, antropométricos e escala analógica visual (EAV) de dor. A coleta do peso e da intensidade da dor foi realizada no período do $6^{\circ}$ ao $9^{\circ}$ mês gestacional, onde a gestante era informada sobre a classificação da escala e orientada a marcá-la de acordo com sua dor.

RESULTADOS: No gráfico comparativo das médias obteve-se um aumento significativo do peso e índice de massa corpórea (IMC) $(\mathrm{p}<0,001)$; a comparação entre o peso e a intensidade da dor lombar no $6^{\circ}$ e $9^{\circ}$ mês, foi encontrada uma correlação de Pearson fraca $(r=0,307)$ e não significativa $(\mathrm{p}>0,05)$ no $6^{\circ}$ mês e uma correlação moderada $(r=0,452)$ e significativa $(p<0,05)$ no $9^{\circ}$ mês. Comparando o peso ponderal com a dor lombar no $9^{\circ}$ mês de gestação, encontrou-se uma correlação

1. Bacharel em Fisioterapia pela Faculdade Integral Diferencial (FACID). Teresina, PI, Brasil.

2. Especializando em Fisioterapia Cardiorrespiratória (CEUT) e Bacharelando em Estatística pela Universidade Federal do Piauí (UFPI). Teresina. PI, Brasil.

3. Mestranda em Alimentos e Nutrição da Universidade Federal do Piauí (UFPI) e Professora de Nutrição da Faculdade Integral Diferencial (FACID). Teresina, PI, Brasil.

Endereço para correspondência:

Cynthia Maria de Sousa Barbosa

Avenida Coronel Costa Araújo, 923

64049-460 Teresina, PI.

Fone: (86) 8864-9789

E-mail: cynthia_barbosa16@hotmail.com moderada $(r=0,532)$ e significativa $(\mathrm{p}<0,05)$ entre as variáveis comparadas.

CONCLUSÃO: Houve correlação entre o aumento ponderal e a intensidade da dor lombar nas gestantes.

Descritores: Dor lombar, Fisioterapia, Ganho de peso, Gravidez.

\section{SUMMARY}

BACKGROUND AND OBJECTIVES: There is increase in energetic supply during gestation to meet both mother and fetus needs, being low back pain a characteristic symptom of this period. This study aimed at correlating weight gain during gestation and low back pain intensity

METHOD: A questionnaire was developed for pregnant women with socio-demographic and anthropometric data, and the pain visual analog scale (VAS). Weight and pain intensity were measured from the $6^{\text {th }}$ to the $9^{\text {th }}$ gestational month, and pregnant women were informed about the scale classification and were oriented to score it according to their pain.

RESULTS: The comparative chart of averages has shown significant weight and body mass index (BMI) gains $(\mathrm{p}<0.001)$; the comparison between weight and pain intensity in the $6^{\text {th }}$ and $9^{\text {th }}$ months has shown a weak ( $\mathrm{r}$ $=0.307)$ and non-significant $(\mathrm{p}>0.05)$ Pearson's correlation in the $6^{\text {th }}$ month, and a moderate $(r=0.452)$ and significant $(p<0.05)$ correlation in the $9^{\text {th }}$ month. When comparing pain to low back pain in the ninth month of gestation, there has been a moderate $(r=0.532)$ and significant $(\mathrm{p}<0.05)$ correlation between variables.

CONCLUSION: There has been correlation between weight gain and low back pain intensity in pregnant women.

Keywords: Low back pain, Physical therapy, Pregnancy, Weight gain. 


\section{INTRODUÇÃO}

O período gestacional é caracterizado por apresentar alterações físicas e emocionais, com o intuito de adaptar a mulher à sua nova condição de gestante ${ }^{1}$. Entre essas modificações estão alterações hormonais, musculoesqueléticas, cardiovasculares, respiratórias, tegumentares, nervosas, gastrintestinais e urogenitais. Estas alterações são fundamentais para regular o metabolismo materno, ajudar no crescimento fetal e preparar a mulher para o momento de trabalho de parto e para a lactação $0^{1,2}$.

O estado nutricional materno está relacionado com as demandas nutricionais, dada pela ingestão de macronutrientes e micronutrientes ${ }^{2}$. $\mathrm{O}$ aumento do aporte energético é necessário para satisfazer as necessidades da mãe e do feto, sendo importante o controle constante do ganho de peso. Sem um suprimento adequado de alimentos e nutrientes poderá comprometer o bem estar de ambos ${ }^{3}$.

$\mathrm{O}$ feto e seus anexos estão presentes na região anterior da pélvis, formando um abdômen protruso e com isso a gestante sofrerá algumas modificações posturais. Observa-se o deslocamento do centro de gravidade, além da liberação dos hormônios (estrógeno e relaxina) que ocasionam em afrouxamento dos ligamentos. A mulher se adaptará mantendo uma nova postura forçando um aumento da curvatura lombar e sobrecarregando os músculos lombares e posteriores da coxa, gerando um processo doloroso ${ }^{4}$.

Com essas alterações surge a queixa mais comum durante gestação, a dor lombar, sendo conceituada por apresentar dor localizada na região inferior do dorso, em uma área situada entre o último arco costal e a prega glútea ${ }^{5}$.

Portanto, o objetivo deste estudo foi correlacionar o ganho de peso ponderal durante a gestação e a intensidade da dor lombar das gestantes.

\section{MÉTODO}

Após aprovação pelo Comitê de Ética e Pesquisa da Faculdade Integral Diferencial sob protocolo $\mathrm{n}^{\mathrm{o}}$ $154 / 10$, realizou-se este estudo do tipo não aleatório, um hospital filantrópico da periferia de Teresina, PI, em que as gestantes foram orientadas e informadas sobre a finalidade da pesquisa e convidadas a assinar o Termo de Consentimento Livre e Esclarecido. O questionário foi respondido individualmente, na sala de espera, nos dias da consulta pré-natal e a seguir era feita a coleta do peso e a intensidade da dor dos meses subsequentes.

O peso pré-gestacional, a altura e a data da última menstruação foram coletadas da cartilha da gestante. Utilizou-se uma balança com carga máxima de $150 \mathrm{~kg}$, para avaliar o peso das gestantes do $6^{\circ}$ ao $9^{\circ}$ mês. Antes de cada pesagem a balança era zerada, a gestante sem calçado e com roupa leve era pesada. A dor na região lombar foi avaliada pela escala analógica visual (EAV), após ser explicado à gestante o significado da escala, sendo assim orientada a marcá-la de zero a 10 nos encontros seguintes.

Foram empregados como critérios de inclusão: gestantes com período gestacional do $6^{\circ}$ ao $9^{\circ}$ mês, pois no primeiro trimestre há ocorrência de vômitos e náuseas que podem se agravar levando à hiperemese gravídica que interfere no ganho ponderal e com altura média entre 1,55 e $1,70 \mathrm{~m}$.

Os critérios de exclusão foram adolescentes por ainda estarem em fase de crescimento; mulheres com deficiência motora e com idade superior a 40 anos, por apresentar um quadro álgico intenso devido à deficiência, gestação gemelar, gestantes hipertensas por apresentar retenção hídrica.

Após a coleta dos dados, utilizou-se o programa Statistical Package for the Social Sciences (SPSS ${ }^{\circledR}$, versão 17.0) para análise estatística descritiva. Na análise dos dados, primeiro foi realizado teste de Shapiro-Wilk com objetivo de avaliar a normalidade das variáveis quantitativas. Para verificar a diferença entre as médias realizou-se o teste $t$ Student para amostras emparelhadas e para verificar o grau de associação utilizou-se o coeficiente de correlação de Pearson. Todos os testes com nível de significância estatística de $95 \%(\mathrm{p}<0,05)$.

\section{RESULTADOS}

Das gestantes que participaram da pesquisa, de acordo com as características sociodemográficas, $90 \%$ eram casadas/amasiadas e 10\% solteiras. Quanto à profissão, 5\% auxiliar administrativo, $80 \%$ donas de casa, $5 \%$ passadeira, $5 \%$ doméstica e $5 \%$ administradora. Quanto ao grau de escolaridade, $45 \%$ possuíam o $1^{\circ}$ grau, $50 \%$ o $2^{\circ}$ grau e $5 \%$ o ensino superior. Quanto às doenças prévias, 30\% sentiam dor lombar antes da gestação e $70 \%$ não referiam dor lombar antes da gestação.

A média de idade foi de 27,0 $\pm 5,2$ anos, com o mínimo de 21 anos e máximo de 37 . A idade gestacional 
média foi de 23,25 $\pm 1,19$ semanas com mínima de 21 semanas e máxima de 25 semanas. A média de gestações anteriores foi de $2,10 \pm 1,12$, com no mínimo 1 e máximo de 4 e em consideração a altura a média foi de 1,58 $\pm 0,28$ metros com o mínimo de $1,55 \mathrm{~m} \mathrm{e}$ máximo de $1,64 \mathrm{~m}$. Todos seguiram o padrão de normalidade $(\mathrm{p}>0,05)$, exceto o número de gestação ( $\mathrm{p}$ $=0,003)$ e a altura $(\mathrm{p}=0,02)$.

O gráfico 1 compara as médias do peso $(\mathrm{kg})$ e do índice de massa corpórea (IMC) $\left(\mathrm{kg} / \mathrm{m}^{2}\right)$ antes da gestação e no $9^{\circ}$ mês gestacional, podendo se observar que houve aumento de peso significativo e consequentemente do IMC, no final da gestação comparado com o início $(\mathrm{p}<0,001)$.

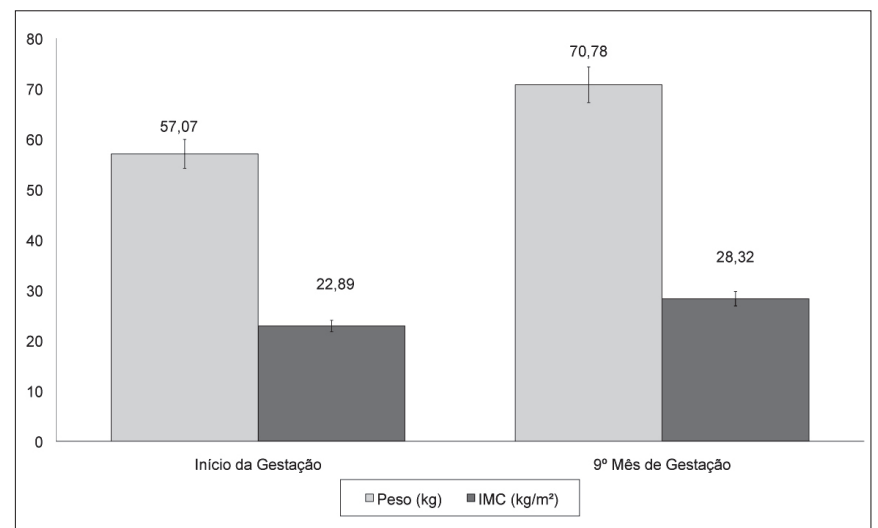

Gráfico 1 - Comparativo entre as médias do peso e do índice de massa corpórea antes e no $9^{\circ}$ mês de gestação.

$* \mathrm{p}<0,001$ : diferença estatisticamente significante antes e no final.

Encontrou-se pequena correlação de Pearson $(r=0,307)$ e não significativa $(p>0,05)$ entre as variáveis peso e a intensidade da dor lombar ao $6^{\circ}$ mês. Já entre o peso ao $9^{\circ}$ mês e a intensidade da dor nesse período, observou-se correlação moderada $(\mathrm{r}=0,452)$ e significativa $(\mathrm{p}<$ $0,05)$. Em relação ao peso ponderal com a dor lombar aos 9 meses de gestação encontrou-se uma correlação moderada $(r=0,532)$ e significativa $(p<0,05)$ entre as variáveis comparadas (Tabela 1$)$.

\section{DISCUSSÃO}

Estudos mostram concordâncias de alguns dados presentes na pesquisa com a dor lombar na gestação, pois a lombalgia gestacional muitas vezes está relacionada à gravidez, e o risco relativo das gestantes em apresentar dores lombares é mais alto do que em mulheres não grávidas. Afirmam ainda que as mulheres que sentiam dor lombar pré-gestacional têm maior probabilidade de apresentarem este quadro álgico durante a gestação ${ }^{6,7}$. Outro estudo evidenciou que a dor lombar pré-gestacional não está relacionada com o aparecimento ou agravamento desta dor ${ }^{8}$.

O tipo e a intensidade de atividades doméstica e laboral realizadas pelas gestantes podem estar relacionadas com o aparecimento da dor lombar. Estudo feito com 45 gestantes houve intensificação da dor com a realização de atividades domésticas. Isso se explica pela sobrecarga imposta associada ao fato de ocorrerem modificações durante a gestação que afetam a biomecânica corporal ${ }^{6}$.

Com relação aos fatores de risco para se desenvolver lombalgia durante a gravidez existem controvérsias na literatura. A idade materna, número de gestações, idade gestacional e peso fetal são alguns dos fatores de risco relacionados com a ocorrência deste sintoma ${ }^{5}$.

Alguns autores afirmam que gestantes mais velhas sentem menos dor que gestantes mais novas e, numa segunda gravidez, a tendência da dor ser mais forte é menor ${ }^{9,11}$. Estudo evidenciou que o pico de maior prevalência ocorreu a partir do $5^{\circ}$ mês de gestação ${ }^{11}$, porém outro estudo afirma que o relato da dor pode ocorrer em qualquer etapa do período gestacional, porém é marcante entre o $4^{\circ}$ e o $7^{\circ}$ mês ${ }^{12}$.

Corroborando com estudo realizado na cidade de Paulínia, SP sobre a prevalência de algias na coluna vertebral durante a gravidez, mostrou que aproximadamente $80 \%$ relataram dores na coluna vertebral e pelve, sendo que $51 \%$ das gestantes com idade gestacional entre 34 e 37 semanas apresentaram dor que interferia significativamente em suas habilidades físicas e qualidade de vida ${ }^{8}$.

Tabela 1 - Correlação de Pearson entre o peso e a intensidade da dor no $6^{\circ}, 9^{\circ}$ mês de gestação e peso ponderal das gestantes do estudo.

\begin{tabular}{lccc}
\hline \multirow{2}{*}{ Categorias } & \multicolumn{2}{c}{ Peso versus Intensidade da Dor } \\
\cline { 2 - 4 } & $6^{\circ}$ Mês de Gestação & $9^{\circ}$ Mês de Gestação & Diferença do Início e ao $9^{\circ}$ Mês de Gestação \\
\hline $\mathrm{p}$ & 0,188 & $0,046^{*}$ & $0,03^{*}$ \\
Correlação de Pearson & 0,307 & $0,452^{* *}$ & $0,532^{* *}$ \\
\hline
\end{tabular}

$* \mathrm{p}<0,05$ : significante; **Correlação moderada 
Esse quadro de dor na região lombar com a evolução da gravidez pode ser explicado porque ocorre um aumento do volume abdominal e o crescimento uterino durante os meses de gestação, ocasionando o deslocamento do centro de gravidade associado a somatório de causas hormonais, mecânicas e vasculares ${ }^{6,13}$.

Pode-se afirmar também que durante a gestação houve um ganho ponderal comparando o peso inicial com o final. O ganho de peso expressa tanto o crescimento fetal quanto a expansão de tecidos maternos placenta, tecido adiposo, útero e mamas, o aumento de líquido extracelular e do volume sanguíneo, além da formação de líquido amniótico ${ }^{14}$.

Observando os dados do presente estudo percebe-se que o ganho ponderal influenciou na intensidade da dor lombar principalmente no $9^{\circ}$ mês de gestação, corroborando dados de estudo que evidencia que a dor lombar mecânica ocorre no período gestacional devido ao aumento generalizado de peso corporal ${ }^{13}$, porém contrapondo a essas afirmações outro estudo afirma não haver correlação entre ganho de peso e dor lombar na gestação ${ }^{15}$.

Observou-se escassez na literatura de pesquisas referentes à relação do ganho ponderal e a intensidade da dor lombar, sendo possível ainda a percepção da importância da atuação da fisioterapia frente a esta sintomatologia que é frequente no período gestacional utilizando-se tratamentos eficazes.

\section{CONCLUSÃO}

Os dados obtidos permitiram evidenciar que houve um ganho de peso concomitante ao tempo de gestação, e que houve correlação entre o aumento ponderal e a intensidade da dor lombar nas gestantes e o aumento da intensidade da dor lombar foi mais significativo no $9^{\circ}$ mês gestacional.

\section{REFERÊNCIAS}

1. Santos MMD, Gallo AP. Lombalgia gestacional: prevalência e características de um programa pré-natal. Arq Bras Ciên Saúde 2010;35(3):174-9.

2. Pitangui ACR, Ferreira CHJ. Avaliação Fisioterapêutica e tratamento da lombalgia gestacional. Fisioter Mov 2008;21(2):135-42.
3. Martins RF, Silva JLPE. Tratamento da lombalgia e dor pélvica posterior na gestação por um método de exercícios. Rev Bras Ginecol Obstet 2005;27(Suppl 5):275-82.

4. Carvalho YBR, Caromano FA. Alterações morfofisiológicas relacionadas com lombalgia gestacional. Arq Ciên Saúde Unipar 2001;5(3):267-72.

5. Ferreira CH, Nakano AM. Conceptual bases supporting the obtention of knowledge about back pain in pregnancy. Rev Lat Am Enfermagem 2001;9(3):95-100.

6. Moura RSV, Campos RS, Mariani SHV, et al. Dor lombar gestacional: impacto de um protocolo de fisioterapia. Arq Med ABC 2007;32(Suppl 2):S59-63.

7. Olsson C, Nilson-Wikmar L. Health-related quality of life and physical ability among pregnant women with and without back pain in late pregnancy. Acta Obstet Gynecol Scand 2004;83(4):351-7.

8. Martins RF, Silva JL. Back pain is a major problem for many pregnant women. Rev Assoc Med Bras 2005;51(3):144-7.

9. Novaes FS, Shimo AKK, Lopes MHBM. Lombalgia na gestação. Rev Latino-Am Enfermagem 2006;14(4):620-4. 10. Batista DC, Chiara VL, Gugelmin AS, et al. Atividade física e gestação: saúde da gestante não atleta e crescimento fetal. Rev Bras Saúde Mater Infant Recife 2003;3(2): 151-8.

11. Toracio MAPC, Korelo RIG. Influência da articulação sacroilíaca na lombalgia gestacional e/ou dor pélvica posterior. Revista Fisioterapia em Evidencia 2010;1(2):5-11.

12. Norén L, Ostgaard S, Johansson G, et al. Lumbar back and posterior pelvic pain during pregnancy: a 3 year follow-up. Eur Spine J 2002;11(3):267-71.

13. Ferreira CHJ, Nakano AMS. Lombalgia na gestação: etiologia, fatores de risco e prevenção. Femina 2000;28(8):435-8.

14. Melo ASO, Assunção PL, Gondim SSR et al. Estado nutricional materno, ganho de peso gestacional e peso ao nascer. Rev Bras Epidemiol 2007;10(2):249-57.

15. Sperandio FF, Santos GM, Pereira F. Características e diferenças da dor sacroilíaca e lombar durante a gestação em mulheres primigestas e multigestas. Rev Fisio Brasil 2004;5(4):267-70.

Apresentado em 06 de junho de 2011.

Aceito para publicação em 31 de agosto de 2011. 